\title{
Aiding Tomorrow's Augmented Cognition Researchers Through Modeling and Simulation Curricula
}

\author{
Julie Drexler, Randall Shumaker, Denise Nicholson, and Cali Fidopiastis \\ Institute for Simulation and Training, University of Central Florida, \\ 3100 Technology Drive, Orlando, FL 32826 \\ \{jdrexler, shumaker, dnichols, cfidopia\}@ist.ucf.edu
}

\begin{abstract}
Research in the newly emerged field of Augmented Cognition (AugCog) has demonstrated great potential to develop more intelligent computational systems capable of monitoring and adapting the systems to the changing cognitive state of human operators in order to minimize cognitive bottlenecks and improve task performance. As the AugCog field rapidly expands, an increasing number of researchers will be needed to conduct basic and applied research in this burgeoning field. However, due to its multidisciplinary nature and cutting-edge technological applications, most traditional academic disciplines cannot support the training needs of future AugCog researchers. Accordingly, an established Modeling and Simulation (M\&S) graduate curriculum is described, which provides a broad basis of interdisciplinary knowledge and skills as well as depth of knowledge within a specific area of the M\&S field. Support for use of the flexible M\&S curriculum to provide the requisite multifaceted foundational training in Augmented Cognition principles is also presented.
\end{abstract}

Keywords: Augmented Cognition, Modeling, Simulation, Cognitive Neuroscience, Adaptive Technology, Human-Computer Interaction, Curriculum Development.

\section{Introduction}

A global trend in $21^{\text {st }}$ century corporate, military, healthcare, and educational settings is the demand to do more with less, which translates into requirements for more efficient selection and assessment of personnel, decreased training time, and reduced manpower. Technological advances have also caused a shift in the tasks of many system operators from primarily physical work to primarily mental work [11]. Moreover, advances in computational capabilities have dramatically increased the prevalence of computers in today's society by providing faster access to increased quantities and types of information available to users. Well-documented limitations in human information processing capabilities (e.g., attention, working memory, sensory input, comprehension, etc.), however, can influence human-system interaction and ultimately impede or even degrade performance [18]. Additionally, the cognitive demands imposed on system users varies as a function of task demands such as the number of tasks that must be simultaneously performed, time constraints 
of the $\operatorname{task}(\mathrm{s})$, level of accuracy required, and even environmental factors such as noise level or temperature [16]. Consequently, there is tremendous need for intelligent technological solutions to improve human systems so that optimal performance can be achieved.

\section{Augmented Cognition}

As previously mentioned, the prevalence of computational systems and interface complexity have dramatically increased over the years, but limitations in the cognitive abilities of human users can impede optimal performance of these systems. In response to the need for more intelligent computational systems, research in the burgeoning field of Augmented Cognition (AugCog) is focused on investigating and developing computational methods, technologies, and non-invasive neurophysiological tools that can be used to accommodate the changing cognitive state of system users in order to improve their performance [18]. Specifically, research in the AugCog field has demonstrated great potential to develop the requisite advanced computational system technologies which can monitor and accommodate the cognitive state of system users by augmenting (i.e., adapting) the system interface to minimize or eliminate information processing bottlenecks inherent in human-system interactions.

\subsection{Augmented Cognition Systems}

Augmented Cognition systems typically function within a closed-loop to manage information processing workload and reduce cognitive bottlenecks associated with human-system interaction applications [4, 19]. A basic closed-loop augmented system contains at least four main components: an operational or simulated environment, automated physiological sensors to monitor and assess a user's cognitive state, an adaptive interface, and an underlying computational architecture which integrates the other three components $[1,3,14]$. Figure 1 presents a graphical representation of a closed-loop augmented cognition system.

Within the closed-loop system shown in Figure 1: (a) the user's senses are stimulated by content and an environment, either in an operational environment by real signals or in a virtual environment by simulated signals. The adaptive system interfaces $(b)$ may be a simple, single audio or visual channel up to a full multimodal array of stimuli [21]. To measure the current state of the system user $(c)$ conventional adaptive multimodal systems relied upon behavioral measures to drive system adaptation (c.f. [15]), whereas Augmented Cognition provides a deeper understanding of the user's cognitive state through the use of neurophysiological sensing techniques. Neurophysiological technologies typically used in Augmented Cognition research have included eye tracking, electroencephalographic (EEG) signals, and functional near-infrared (fNIR) imaging, which monitors blood oxygenation and volume changes in the brain $[2,5,8,10,20]$.

The final step in completing the closed-loop augmented cognition system (cf. Figure 1) is to $(d)$ adapt the environment and system interface based on the measured cognitive state to optimally support the user's performance. When an information processing (i.e., cognitive) bottleneck is detected by the neurophysiological sensors, 


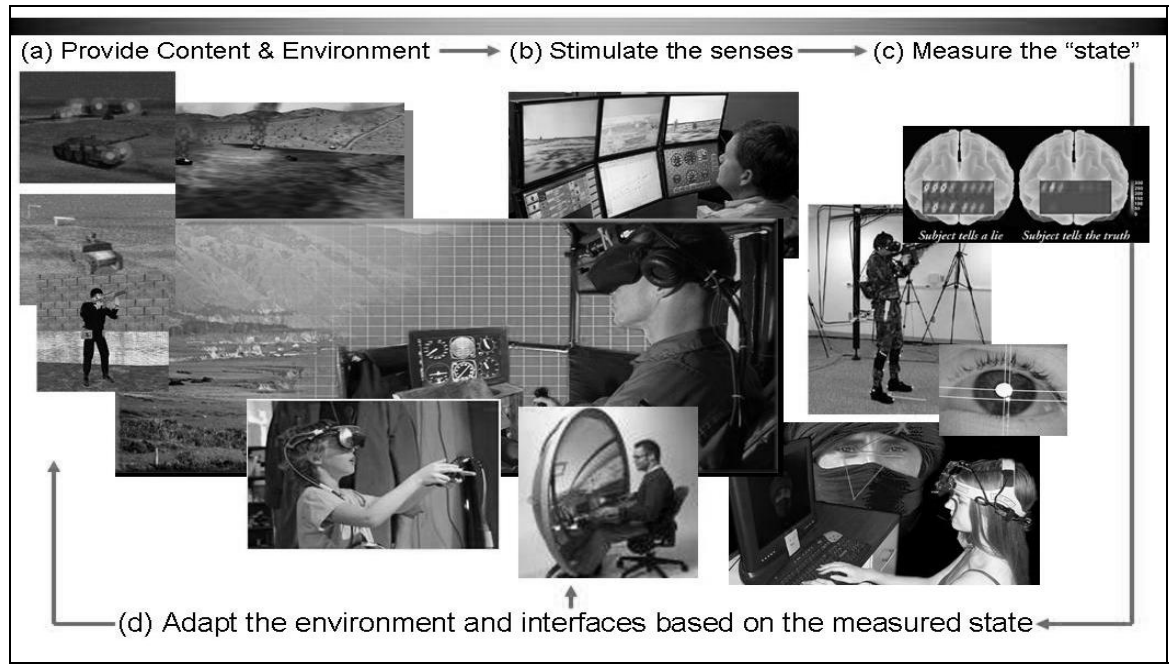

Fig. 1. Closed-loop augmented cognition system

they trigger the selection of mitigation strategies. A mitigation strategy is an intervention technique that leads into a corresponding set of concrete adaptive actions (e.g., attention management, multimodal interface adaptation, modality switching, operational performance support, task cooperation, dynamic task reallocation, and individualized embedded training strategies), which are then conveyed to the system user through an adapted interface [14, 17]. As the adaptation is applied to the interface, updated neurophysiological metrics of the operator's cognitive state become available. Thus, the Augmented Cognition system can iteratively evaluate the success of a given mitigation and initiate further mitigating actions if problems persist, and thus "close the loop" around the system user [14, 19].

\subsection{Research in Augmented Cognition}

The preceding discussion highlights the fact that the field of Augmented Cognition does not draw from one scientific discipline but, rather is an interaction and collaboration of multidisciplinary areas including cognitive science, neuroscience, cognitive psychology, human factors (engineering and psychology), systems engineering, and computer science. Over the past few years, the field has also been able to more solidly establish research methodologies that include state-of-the-art technologies such as functional near-infrared imaging (fNIR). Moreover, a closedloop augmented cognition system could be beneficial for the design and/or evaluation of most any human-operated computational system which requires that the system be adaptable to the changing cognitive needs of the user [19]. Accordingly, the Augmented Cognition field is rapidly expanding and as a result, the demand for researchers qualified to conduct basic and applied research in this emergent field is expected to increase in the future. Due to its multidisciplinary nature and cuttingedge technological applications, however, most traditional academic disciplines cannot support the training needs of future Augmented Cognition researchers. In 
order to prevent what seems to be an imminent shortage of qualified Augmented Cognition professionals, it is imperative to identify the core competencies needed by future researchers and develop an academic program to provide the educational training these future professionals require to succeed in their careers.

\section{Modeling and Simulation}

The issues involving the preparation of future Augmented Cognition researchers are remarkably similar to the issues faced by the professional and academic members of the established Modeling and Simulation (M\&S) community when it first emerged as a new multidisciplinary field. Specifically, the rapid advancement of computer-aided training systems in the early 1990's exposed the need to develop a professional educational training pathway for unskilled persons filling vacancies within the diverse simulation job market. Ensuing debates on appropriate curricula led to the creation of an interdisciplinary program of study, which exposed students to a cross-section of seemingly disparate subject matter such as Psychology, Engineering, and Computer Science. Although, when this information was applied collectively within an integrated curriculum, a graduate of this type of program was capable of flexibly solving problems within a multitude of occupations including those involving humancentered design.

\subsection{Interdisciplinary Graduate Program in Modeling and Simulation}

Over the years, academic institutions have progressed from providing certification level curricula to supporting accredited doctoral degree programs in Modeling and Simulation (M\&S). The University of Central Florida (UCF) is one of the first universities to offer interdisciplinary graduate programs in Modeling and Simulation; other M\&S graduate programs include the Naval Post-Graduate School, Old Dominion University, and Georgia Institute of Technology [9]. As with the field of Augmented Cognition, M\&S practitioners require both generalized and specialized skills due to the size and complexity of the field [22]. Therefore, industry and government leaders in the M\&S community actively participated in the design of the $M \& S$ curriculum and provided critical input regarding the broad foundation of interdisciplinary skills (i.e., the knowledge and relevant tools) that were needed by $\mathrm{M} \& \mathrm{~S}$ practitioners as well as input on specific knowledge required to work within the various areas of the M\&S field [9].

UCF's Modeling and Simulation curriculum for the master's degree is comprised of two main components. One component consists of a standardized set of courses designed to provide an interdisciplinary core body of knowledge in the fundamentals of $M \& S$ : Introduction to $M \& S$, Quantitative Aspects of M\&S, Engineering Statistics, Advanced Research Methodology, and Simulation Research Methods and Practicum. In addition to learning the M\&S fundamentals, these courses are also intended to aid the development of a student's ability to critically review the scientific literature in the $M \& S$ field, solve complex problems through building simulation models, designing and carrying out experiments, analyzing data, communicating their findings, and manage M\&S programs [22]. The other main curriculum component 
enables students to establish depth of knowledge within a specific area of the M\&S field. UCF's interdisciplinary graduate program offers seven different M\&S focus areas, which vary in terms of the required cornerstone courses and restricted electives in the curriculum. An overview of each focus area (brief description, cornerstone course, typical electives, and discipline areas from which the courses are drawn) is provided below [22].

Quantitative Aspects of Simulation: Focuses on applying advanced quantitative methods to modeling and simulation. Prepares graduates to apply mathematics and statistics to build multidisciplinary models and simulations. Cornerstone course: Mathematical Modeling. Typical electives: Statistical Aspects of Digital Simulation, Advanced Systems Simulation, Optimization Theory, and Data Fitting. Curriculum disciplines: Electrical Engineering, Mechanical Engineering, Industrial/Systems Engineering, Applied Mathematics, and Statistics.

Simulation Infrastructure: Focuses on an in-depth understanding of simulation systems' components and patterns of configuration and communication. Prepares graduates to develop and implement distributed simulation and training environments and/or manage a team developing these types of systems. Cornerstone course: Performance Models of Computers and Networks. Typical electives: High Performance Computer Architecture, Performance Analysis of Computer and Communication Systems, Computer Communication Networks Architecture. Curriculum disciplines: Computer Design/Architecture, Computer Programming, Computing Theory, Electrical Engineering, and Information Systems Management.

Simulation Managemen: Focuses on the management of modeling, simulation, and training (MS\&T) projects. Prepares graduates to manage MS\&T projects for military agencies or companies. Cornerstone course: The Environment of Technical Organizations; Typical electives: Simulation-Based Life cycle Engineering, Management Information Systems, Project Engineering, and Engineering Management. Curriculum disciplines: Industrial Engineering; Industrial/Systems Engineering; Electrical Engineering; and Mechanical Engineering.

Computer Visualization in $M \& S$ : Focuses on the technical aspects of computer graphic systems, virtual environments, and human-centered simulation systems. Prepares graduates to apply the state-of-the-art in computer graphics and other human-interface technologies. Cornerstone course: Computer Graphics. Typical electives: Computer Vision Systems, Engineering Applications of Computer Graphics, Machine Perception, and Human-Computer Interaction. Curriculum disciplines: Computer Applications for Computer Scientists, Electrical Engineering, and Industrial Engineering.

Simulation Modeling and Analysis: Focuses on the effective use of simulation as a tool for designing, planning, analysis, and decision making with an emphasis on problem definition, model formulation, design of simulation experiments, and modelbased analysis. Prepares graduates to work with corporate and government decision makers in modeling and evaluating the impacts of proposed policies and system designs. Cornerstone course: Discrete Systems Simulation. Typical electives: Simulation Design and Analysis, Object-Oriented Simulation, Experimental Design, 
and Advanced Systems Simulation. Curriculum disciplines: Electrical Engineering, Industrial Engineering, and Industrial/Systems Engineering.

Interactive Simulation/Intelligent Systems: Focuses on the basic tools for creating designs for simulators and simulator-based training systems and the application of expert systems and other intelligent systems in a simulation setting. Prepares graduates for a career in the training simulation/simulator industries. Cornerstone course: Interactive Simulation. Typical electives: Training Systems Design, Intelligent Simulation, and Expert Systems and Knowledge Engineering. Curriculum disciplines: Computer Applications for Computer Scientists, Electrical Engineering, Industrial Engineering, Education: Technology and Media, and Transportation Engineering.

Human Systems in $M \& S$ : Focuses on the content and techniques of human behavior in simulation systems (e.g., human factors, human-computer interaction, virtual worlds, statistical and quantitative procedures, experimental design, and other research methodologies). Prepares graduates for research and development work on topics such as human-in-the-loop simulation and the use of various sensory input/output modalities to coordinate human-machine activities. Cornerstone course: Human Factors or Usability Engineering. Typical electives: Human-Computer Interaction, Human Performance, Advanced Research Methodology, Human Cognition and Learning. Curriculum disciplines: Industrial Engineering, Education: Technology and Media, Experimental Psychology, Industrial and Applied Psychology, Psychology; and Digital Media.

The curriculum for a doctoral degree expands on the master's degree requirements. In addition to adding Human-Computer Interaction to the set of required interdisciplinary core course, the Ph.D. curriculum incorporates a second set of standard interdisciplinary courses, called a "Restricted Core", which requires students to complete at least two courses from the following courses: Mathematical Modeling; Interactive Simulation or Continuous Simulation; and Discrete Systems Simulation or Object-Oriented Simulation [22]. The doctoral curriculum also requires students to complete at least three of the seven M\&S focus area cornerstone courses.

Students in the M\&S graduate program at UCF also have opportunities to obtain practical research and development $(\mathrm{R} \& \mathrm{D})$ experience in any of the seven focus areas. One type of opportunity available to students is to work with members of the $M \& S$ faculty on R\&D projects. Central Florida Research Park, located adjacent to UCF's main campus, is the hub of modeling, simulation and training research and acquisition for the Department of Defense [7]. Accordingly, other potential R\&D opportunities include: The Naval Air Warfare Center, Training Systems Division (NAVAIR TSD); Air Force Agency for Modeling and Simulation; U.S. Army Program Executive Office for Simulation, Training and Instrumentation (PEO STRI); US Army Research Development and Engineering Command Simulation and Training Technology Center (RDECOM STTC); Marine Corps Program Manager for Training Systems (PMTRASYS), the Army Research Institute (ARI) Human Research and Engineering Directorate, and the Coast Guard Liaison Office; or at one of more than $140 \mathrm{M} \& \mathrm{~S}$ related companies that have offices in the local area. 


\section{Applying an M\&S Curriculum for Training Augmented Cognition Researchers}

As discussed previously, the multi-disciplinary field of Augmented Cognition recently emerged in response to the need for computational systems that could monitor the cognitive state of human operators and adapt the system to their changing needs in order to minimize cognitive bottlenecks and improve task performance. To date, Augmented Cognition research has been primarily sponsored by military and defense agencies, but the commercial sector has shown interest in developing Augmented Cognition systems to manage information overload for non-military applications (e.g., air traffic control; [1]). Future applications of Augmented Cognition systems have also been proposed for education and training areas such as intelligent tutoring [13], synthetic learning environments [12], skills assessment (e.g., military selection and classification), and cognitive rehabilitation [6]. Consequently, as the Augmented Cognition field expands, an increasing number of researchers will be needed to work on basic and applied R\&D projects.

The M\&S graduate curriculum, described in the previous section, incorporates a broad basis of interdisciplinary knowledge and skills, many of which are also required to conduct effective research in the Augmented Cognition field. Accordingly, the

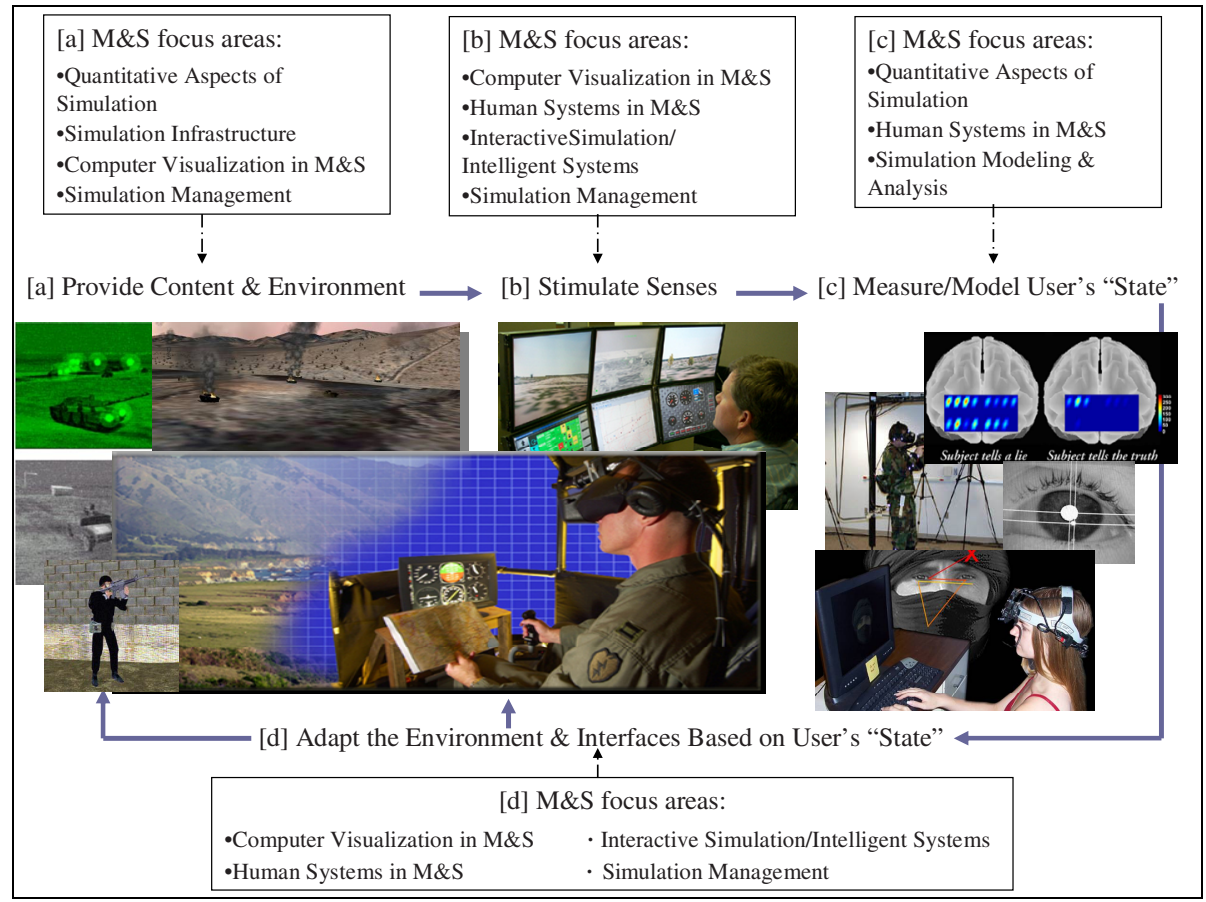

Fig. 2. M\&S focus areas mapped to an augmented cognition closed-loop system 
existing M\&S graduate curriculum could be used to provide the foundation of diverse educational requirements that would enable future Augmented Cognition professionals to conduct basic and applied research in a variety of areas. Figure 2 provides a graphical representation of the relationship between the key components of an Augmented Cognition system and the various focus areas of the M\&S curriculum.

The information shown in Figure 2 suggests that the various focus areas of the M\&S curriculum provide the multifaceted approach necessary to train future augmented cognition researchers. Thus, the mature, yet flexible curriculum of a Modeling and Simulation program could offer the proper melding of psychology and technology-based coursework to support foundational training in Augmented Cognition principles as well as proving an opportunity for hands-on experience in research domains associated with the field.

\section{References}

1. Augmented Cognition International Society: What is augmented cognition? Retrieved January 30, 2007 from http://www.augmentedcognition.org/

2. Berka, C., Levendowski, D.J., Davis, G., Lumicao, M.N., Ramsey, C.K., Stanney, K., Reeves, L., Tremoulet, P.D., Harkness Regli, S.: EEG Indices Distinguish Spatial and Verbal Working Memory Processing: Implications for Real-Time Monitoring in a ClosedLoop Tactical Tomahawk Weapons Simulation. In: Schmorrow, D.D. (ed.) Foundations of Augmented Cognition, pp. 405-413. Lawrence Erlbaum Associates, Mahwah (2005)

3. Dickson, B.T.: Closed Loop Systems - Stability and Predictability. In: Schmorrow, D.D. (ed.) Foundations of Augmented Cognition, pp. 617-620. Lawrence Erlbaum Associates, Mahwah (2005)

4. Dorneich, M.C., Whitlow, S.D., Mathan, S., Carciofini, J., Ververs, P.M.: The Communications Scheduler: A Task Scheduling Mitigation for a Closed Loop Adaptive System. In: Schmorrow, D.D. (ed.) Foundations of Augmented Cognition, pp. 132-141. Lawrence Erlbaum Associates, Mahwah (2005)

5. Downs III, H.D., Downs, D., Robinson, W., Nishimura, E., Stautzenberger, J.P.: A New Approach to fNIR: The Optical Tomographic Imaging Spectrometer. In: Schmorrow, D.D. (ed.) Foundations of Augmented Cognition, pp. 205-206. Lawrence Erlbaum Associates, Mahwah (2005)

6. Fidopiastis, C.M., Stapleton, C.B., Whiteside, J.D., Hughes, C.E., Fiore, S.M., Martin, G.A, Rolland, J.P., Smith, E.M.: Human Experience Modeler: Context-Driven Cognitive Retraining to Facilitate Transfer of Learning. Cyberpsychol. Behav. 9, 183-187 (2006)

7. Institute for Simulation and Training. Retrieved February 6, 2007 from http://www.ist.ucf.edu

8. Izzetoglu, M., Shoko, N., Bunce, S., Izzetoglu, K., Onaral, B., Chance, B.: Hemodynamic Response Estimation During Cognitive Activity Using fNIR Spectroscopy. In: Schmorrow, D.D. (ed.) Foundations of Augmented Cognition, pp. 207-209. Lawrence Erlbaum Associates, Mahwah (2005)

9. Kincaid, J.P., Hamilton, R., Tarr, R.W., Sangani, H.: Simulation in Education and Training. In: Obaidat, M.S., Papadimitriou, G.I. (eds.) Applied System Simulation: Methodologies and Applications, pp. 437-456. Kluwer Academic Publishers, Norwell (2003)

10. May, J.G., Kennedy, R.S., Williams, M.C., Dunlap, W.P., Brannan, J.R.: Eye Movement Indices of Mental Workload. Acta Psychol. 75, 75-89 (1990) 
11. Moroney, W.F., Bittner Jr., A.C.: Military Systems Techniques. In: Weimer, J. (ed.) Research Techniques in Human Engineering, pp. 363-438. Prentice Hall, Englewood Cliffs (1995)

12. Nasoz, F., Alvarez, K., Lisetti, C., Finkelstein, N.: Emotion Recognition from Physiological Signals for Presence Technologies. Int. J. Cogn. Technol. Work - Special Issue on Presence 6, 4-14 (2003)

13. Nicholson, D.M., Lackey, S.J., Arnold, R., Scott, K.: Augmented Cognition Technologies Applied to Training: A Roadmap for the Future. In: Schmorrow, D.D. (ed.) Foundations of Augmented Cognition, pp. 931-940. Lawrence Erlbaum Associates, Mahwah (2005)

14. Nicholson, D., Stanney, K., Fiore, S., Davis, L., Fidopiastis, C., Finkelstein, N., Arnold, R.: An Adaptive System for Improving and Augmenting Human Performance. In: Schmorrow, D.D., Stanney, K.M., Reeves, L.M. (eds.) Foundations of Augmented Cognition. 2nd edn. Strategic Analysis, Inc and the Augmented Cognition International Society, pp. 215-222. Arlington, Virginia (2006)

15. Osga, G.A.: 21st Century Workstations - Active Partners in Accomplishing Task Goals. In: Proceedings of the Human Factors and Ergonomics Society 44th Annual Meeting. Human Factors and Ergonomics Society, Santa Monica (2000)

16. Proctor, R.W., Van Zandt, T.: Human Factors in Simple and Complex Systems. Allyn and Bacon, Boston (1994)

17. Regli, S.H., Tremoulet, P., Hastie, H., Stibler, K.: Mitigation Strategy Design for Optimal Augmented Cognition Systems. In: Schmorrow, D.D., Stanney, K.M., Reeves, L.M. (eds.) Foundations of Augmented Cognition. 2nd edn. Strategic Analysis, Inc and the Augmented Cognition International Society, pp. 208-214. Arlington, Virginia (2006)

18. Schmorrow, D.D., Kruse, A.A.: Augmented Cognition. In: Bainbridge, W.S. (ed.) Berkshire Encyclopedia of Human-Computer Interaction, pp. 54-59. Berkshire Publishing, Great Barrington, Massachusetts (2004)

19. Schmorrow, D.S., Reeves, L.M., Bolton, A.: 21st Century Human Systems Integration: Augmented Cognition for Improved Defense Readiness. Paper presentation at The Technical Cooperation Program (TTCP) Human Resources and Performance Group (HUM) Human Systems Integration (HSI) Symposium on The Science and Practice of HIS for Defence Systems, Russell, Canberra (May 4-5, 2006)

20. St. John, M., Kobus, D.A., Morrison, J.G., Schmorrow, D.: Overview of the DARPA Augmented Cognition Technical Integration Experiment. Int. J. Hum. Comput. Interact. 17, 131-149 (2004)

21. Stanney, K., Samman, S., Reeves, L., Hale, K., Buff, W., Bowers, C., Goldiez, B., Nicholson, D., Lackey, S.: A Paradigm Shift in Interactive Computing: Deriving Multimodal Design Principles from Behavioral and Neurological Foundations. Int. J. Hum. Comput. Interact. 17, 229-257 (2004)

22. University of Central Florida: Graduate Catalog (2006-2007) [On-line]. Available: http://graduate.ucf.edu/catalog/ 